\title{
Asian Option Pricing with Monotonous Transaction Costs under Fractional Brownian Motion
}

\author{
Di Pan, Shengwu Zhou, Yan Zhang, and Miao Han \\ College of Sciences, China University of Mining and Technology, Jiangsu, Xuzhou 221116, China \\ Correspondence should be addressed to Shengwu Zhou; zswcumt@163.com
}

Received 27 September 2013; Accepted 26 November 2013

Academic Editor: Song Cen

Copyright (C) 2013 Di Pan et al. This is an open access article distributed under the Creative Commons Attribution License, which permits unrestricted use, distribution, and reproduction in any medium, provided the original work is properly cited.

Geometric-average Asian option pricing model with monotonous transaction cost rate under fractional Brownian motion was established. The method of partial differential equations was used to solve this model and the analytical expressions of the Asian option value were obtained. The numerical experiments show that Hurst exponent of the fractional Brownian motion and transaction cost rate have a significant impact on the option value.

\section{Introduction}

In 1989, Peters [1] firstly proposed that fractional Brownian motion could be used to describe the changes of asset prices. In 2000, the theory of stochastic integral about fractional Brownian motion was studied by Duncan et al. [2], and fractional Itô's formula and Girsanov theorem under the fractional Brownian motion were derived. The fractional Itô's integral had been further developed by Biagini et al. [3] for $H \geq 1 / 2$. Equivalent definition of fractional Itô's integral was introduced by Alos et al. [4] and Bender [5]. Necula [6] utilized the knowledge of fractal geometry and deduced the Black-Scholes option pricing formula under fractional Brownian motion, which was of great significance to the development of option pricing with fractional Brownian motion. The transaction cost is an important factor affecting the option pricing. Many scholars had studied the pricing problems of contingent claim with transaction costs. Leland [7] groundbreakingly proposed that a modified volatility should be applied to solving the problem of hedging error brought by transaction costs in Black-Scholes model. Barles and Soner [8] assumed that the investor's preference satisfied exponential utility function and provided a more complex model. The Black-Scholes option pricing model with transaction costs was given by Amster et al. [9]. Liu and Chang [10] and Wang et al. [11] studied the European option pricing with transaction costs under the fractional Brownian motion. The pricing studies for the Asian option mostly are based on the standard Brownian motion, but the time-varying of Asian option pricing model under fractional Brownian motion had not been studied systematically.

Based on the previous references, the geometric Asian option pricing model with monotonous transaction rates under the fractional Brownian motion was presented, and the analytic expression of the Asian option value was derived. The influence of Hurst exponent and transaction cost on the Asian option value was discussed through the numerical calculations.

This paper's outline is as follows. In Section 2, we studied the geometric-average Asian option pricing model under the fractional Brownian motion. The closed-form solution of the pricing model was presented in Section 3. In Section 4, the numerical examples were given. Section 5 serves as the conclusion of the whole paper.

\section{Geometric-Average Asian Option Pricing Model for a Fractional Brownian Motion under Monotonous Transaction Cost Rate}

Let $(\Omega, F, P)$ be a complete probability space carrying a fractional Brownian motion $\left\{B_{H}(t), t \geq 0\right\}$ with Hurst exponent 
$H(0<H<1)$, that is, a continuous, centered Gaussian process with covariance function [12]

$$
\begin{aligned}
\operatorname{Cov} & \left(B_{H}(t), B_{H}(s)\right) \\
& =\frac{1}{2}\left(|t|^{2 H}+|s|^{2 H}-|t-s|^{2 H}\right), \quad s, t \in R .
\end{aligned}
$$

If $H=1 / 2$, then the corresponding fractional Brownian motion is the usual standard Brownian motion.

In this paper we will drive a geometric-average Asian option pricing model under the following assumptions.

(i) The price $S_{t}$ of the underlying stock satisfies

$$
d S_{t}=r_{t} S_{t} d t+\sigma S_{t} d B_{H}(t)
$$

where $r_{t}$ is the expected yields, $\sigma$ is the stock price volatility, and $B_{H}(t)$ is the fractional Brownian motion with Hurst exponent.

(ii) The risk-free interest rate $r_{t}$ is a deterministic function of time $t$.

(iii) Suppose $v_{t}$ shares of the underlying stock are bought $\left(v_{t}>0\right)$ or sold $\left(v_{t}<0\right)$ at the price $S_{t}$; then the transaction cost is given by $h\left(v_{t}\right)\left|v_{t}\right| S_{t}$ in either buying or selling; here $h\left(v_{t}\right)=a-b v_{t}(a, b>0)$ is a monotone transaction cost rate.

(iv) The expected return rate of the portfolio equals the risk-free interest rate.

(v) The portfolio is revised every $\delta t$, where $\delta t$ is a finite and fixed small time step.

Let $V=V\left(t, J_{t}, S_{t}\right)$ be the value of the geometric-average Asian call option on the time $t$, where $J_{t}=e^{(1 / t) \int_{0}^{t} \ln S_{\tau} d \tau}$ is the geometric average of the underlying asset price in the time period of $[0, t]$.

Construct a portfolio $\Pi_{t}$ : long position of a Geometricaverage Asian call option and short position of $\Delta_{t}$ shares of underlying asset. Then the value of the portfolio at current time $t$ is

$$
\Pi_{t}=V_{t}-\Delta_{t} S_{t} .
$$

In the period $[t, t+\delta t]$, the change in the value of the portfolio is

$$
\begin{aligned}
\delta \Pi_{t}= & \delta V_{t}-\Delta_{t} \delta S_{t}-\left[\left(a-b\left|v_{t}\right|\right)\left|v_{t}\right| S_{t}\right] \\
= & \left(\frac{\partial V}{\partial t}+H \sigma^{2} S_{t}^{2} t^{2 H-1} \frac{\partial^{2} V}{\partial S_{t}^{2}}\right) \delta t+\left(\frac{\partial V}{\partial S_{t}}-\Delta_{t}\right) \delta S_{t} \\
& +\frac{\partial V}{\partial J_{t}} \delta J_{t}-\left[\left(a-b\left|v_{t}\right|\right)\left|v_{t}\right| S_{t}\right]
\end{aligned}
$$

where

$$
\begin{gathered}
v_{t}=\frac{\partial V}{\partial S_{t+\delta t}}-\frac{\partial V}{\partial S_{t}}=\frac{\partial^{2} V}{\partial S_{t}^{2}} \delta S_{t}+\frac{\partial^{2} V}{\partial S_{t} \partial J_{t}} \delta J_{t} \\
+O(\delta t)=\frac{\partial^{2} V}{\partial S_{t}^{2}} \sigma S_{t} \delta B_{H}(t)+O(\delta t) .
\end{gathered}
$$

Owing to

$$
E\left(a\left|\nu_{t}\right| S_{t}\right)=\sqrt{\frac{2}{\pi}} a \sigma S_{t}^{2}(\delta t)^{H}\left|\frac{\partial^{2} V}{\partial S_{t}^{2}}\right|+O(\delta t),
$$

we have

$$
\begin{aligned}
E\left[b\left|\nu_{t}\right|^{2} S_{t}\right] & \\
= & b \sigma^{2} S_{t}^{3}\left(\frac{\partial^{2} V}{\partial S_{t}^{2}}\right)^{2}\left\{\operatorname{Var}\left(\delta B_{H}(t)\right)-\left[E\left(\delta B_{H}(t)\right)\right]^{2}\right\} \\
& +O\left(\delta t^{2}\right) \\
= & b \sigma^{2} S_{t}^{3}\left(\frac{\partial^{2} V}{\partial S_{t}^{2}}\right)^{2}(\delta t)^{2 H}+O\left(\delta t^{2}\right) ;
\end{aligned}
$$

hence we have

$$
\begin{aligned}
E\left[\left(a-b\left|v_{t}\right|\right)\left|v_{t}\right| S_{t}\right]= & E\left[a\left|v_{t}\right| S_{t}\right]-E\left[b\left|v_{t}\right|^{2} S_{t}\right] \\
= & \sqrt{\frac{2}{\pi}} a \sigma S_{t}^{2}(\delta t)^{H}\left|\frac{\partial^{2} V}{\partial S_{t}^{2}}\right| \\
& -b \sigma^{2} S_{t}^{3}\left(\frac{\partial^{2} V}{\partial S_{t}^{2}}\right)^{2}(\delta t)^{2 H} .
\end{aligned}
$$

By the assumption (iv) we have

$$
E\left(d \Pi_{t}\right)=r \Pi_{t} d t .
$$

And then

$$
\begin{aligned}
r(V & \left.-\Delta_{t} S_{t}\right) \delta t \\
= & \left(\frac{\partial V}{\partial t}+H \sigma^{2} S_{t}^{2} t^{2 H-1} \frac{\partial^{2} V}{\partial S_{t}^{2}}\right) \delta t+\frac{\partial V}{\partial J_{t}} \delta J_{t} \\
& -\sqrt{\frac{2}{\pi}} a \sigma S_{t}^{2}(\delta t)^{H}\left|\frac{\partial^{2} V}{\partial S_{t}^{2}}\right|+b \sigma^{2} S_{t}^{3}\left(\frac{\partial^{2} V}{\partial S_{t}^{2}}\right)^{2}(\delta t)^{2 H}
\end{aligned}
$$

Owing to

$$
J_{t}=e^{(1 / t) \int_{0}^{t} \ln S_{\tau} d \tau}
$$

then

$$
\frac{\delta J_{t}}{\delta t}=\frac{J_{t} \ln \left(S_{t} / J_{t}\right)}{t} .
$$

Choosing $\Delta_{t}=\partial V / \partial S_{t}$ and substituting it into (10), the following formula can be obtained:

$$
\begin{aligned}
\frac{\partial V}{\partial t}+ & H \sigma^{2} S_{t}^{2} t^{2 H-1} \frac{\partial^{2} V}{\partial S_{t}^{2}}+r S_{t} \frac{\partial V}{\partial S_{t}} \\
& -\sqrt{\frac{2}{\pi}} a \sigma S_{t}^{2}(\delta t)^{H-1}\left|\frac{\partial^{2} V}{\partial S_{t}^{2}}\right|+b \sigma^{2} S_{t}^{3}\left(\frac{\partial^{2} V}{\partial S_{t}^{2}}\right)^{2}(\delta t)^{2 H-1} \\
& +\frac{\partial V}{\partial J_{t}} \frac{J_{t} \ln \left(S_{t} / J_{t}\right)}{t}-r V=0 .
\end{aligned}
$$


Let

$$
\begin{aligned}
\operatorname{Le}(H)= & \sqrt{\frac{2}{\pi}} \frac{a}{\sigma}(\delta t)^{H-1}, \\
\widetilde{\sigma}^{2}=2 \sigma^{2}[ & {\left[H t^{2 H-1}-\operatorname{Le}(H) \operatorname{sign}\left(V_{S S}\right)\right.} \\
& \left.+b S_{t} V_{S S}(\delta t)^{2 H-1}\right] .
\end{aligned}
$$

Substituting these into (13), we get the following conclusions.

Theorem 1. If the underlying asset price $S_{t}$ satisfies fractional Brownian motion (2), then the value of Geometric-average Asian call option with monotonous transaction cost rate at the time $t(0 \leq t \leq T)$ satisfies the following mathematical model:

$$
\begin{gathered}
\frac{\partial V}{\partial t}+\frac{1}{2} \widetilde{\sigma}^{2} S_{t}^{2} \frac{\partial^{2} V}{\partial S_{t}^{2}}+r S_{t} \frac{\partial V}{\partial S_{t}}+\frac{\partial V}{\partial J_{t}} \frac{J_{t} \ln \left(S_{t} / J_{t}\right)}{t}-r V=0, \\
V\left(T, J_{T}, S_{T}\right)=\left(J_{T}-K\right)^{+} .
\end{gathered}
$$

Remark 2. For a single European option with long position, the yield at expiration date is $\left(J_{T}-K\right)^{+}$or $\left(K-J_{T}\right)^{+}$. Because they are convex functions, $V_{J J}>0$. Due to $J_{t}=e^{(1 / t) \int_{0}^{t} \ln S_{\tau} d \tau}$, thus $V_{S S}>0$. Then (14) can be represented as

$$
\widetilde{\sigma}^{2}=2 \sigma^{2}\left(H t^{2 H-1}-\sqrt{\frac{2}{\pi}} \frac{a}{\sigma}(\delta t)^{H-1}+b S_{t}(\delta t)^{2 H-1}\right) .
$$

\section{Option Pricing Formulas}

Theorem 3. Supposing the underlying asset prices $S_{t}$ satisfy (2), then at time $t$ the value $V\left(t, J_{t}, S_{t}\right)$ of Geometric-average Asian call option with transaction costs with expiration date $T$ and exercise price $K$ is as follows:

$$
\begin{aligned}
V\left(t, J_{t}, S_{t}\right)= & \left(J_{t}^{t} S_{t}^{T-t}\right)^{(1 / T)} e^{r^{*}(T-t)-\int_{t}^{T} r_{\theta} d \theta+\left(\sigma^{* 2} / 2\right)\left(T^{2 H}-t^{2 H}\right)} \\
& \times N\left(d_{1}\right)-K e^{-\int_{t}^{T} r_{\theta} d \theta} N\left(d_{2}\right)
\end{aligned}
$$

where

$$
\begin{aligned}
d_{1}= & \frac{\ln \left[\left(J_{t}^{t} S_{t}^{T-t}\right)^{1 / T} / K\right]+r^{*}(T-t)+\sigma^{* 2}\left(T^{2 H}-t^{2 H}\right)}{\sigma^{*} \sqrt{T^{2 H}-t^{2 H}}}, \\
d_{2}= & d_{1}-\sigma^{*} \sqrt{T^{2 H}-t^{2 H}}, \\
r^{*}= & \frac{\int_{t}^{T} r_{\theta}((T-\theta) / T) d \theta}{T-t}-\frac{\sigma^{2}\left(T^{2 H}-t^{2 H}\right)}{2(T-t)} \\
& +\frac{H \sigma^{2}\left(T^{2 H+1}-t^{2 H+1}\right)}{T(2 H+1)(T-t)} \\
& +\frac{1}{2} L e(H) \sigma^{2} \frac{T-t}{T}-\frac{1}{2} \sigma^{2} b S_{t}(\delta t)^{2 H-1} \frac{T-t}{T},
\end{aligned}
$$

$$
\begin{aligned}
\sigma^{*}=\sigma(1 & -\frac{4 H\left(T^{2 H+1}-t^{2 H+1}\right)}{T(2 H+1)\left(T^{2 H}-t^{2 H}\right)} \\
& +\frac{H\left(T^{2 H+2}-t^{2 H+2}\right)}{T^{2}(H+1)\left(T^{2 H}-t^{2 H}\right)}-2 L e(H) \\
& \left.\times \frac{(T-t)^{3}}{3 T^{2}\left(T^{2 H}-t^{2 H}\right)}+\frac{2 b S_{t}(\delta t)^{2 H-1}(T-t)^{3}}{3 T^{2}\left(T^{2 H}-t^{2 H}\right)}\right)^{1 / 2}
\end{aligned}
$$

$$
L e(H)=\sqrt{\frac{2}{\pi}} \frac{a}{\sigma}(\delta t)^{H-1}, \quad N(x)=\int_{-\infty}^{x} \frac{1}{\sqrt{2 \pi}} e^{-\left(t^{2} / 2\right)} d t .
$$

Proof. Let

$$
\xi_{t}=\frac{1}{T}\left[t \ln J_{t}+(T-t) \ln S_{t}\right], \quad V\left(t, J_{t}, S_{t}\right)=U\left(t, \xi_{t}\right) ;
$$

then

$$
\begin{aligned}
& \frac{\partial V}{\partial t}=\frac{\ln \left(J_{t} / S_{t}\right)}{T} \frac{\partial U}{\partial \xi_{t}}+\frac{\partial U}{\partial t}, \quad \frac{\partial V}{\partial S_{t}}=\frac{T-t}{T S_{t}} \frac{\partial U}{\partial \xi_{t}}, \\
& \frac{\partial^{2} V}{\partial S_{t}^{2}}=\left(\frac{T-t}{T S_{t}}\right)^{2} \frac{\partial^{2} U}{\partial \xi_{t}^{2}}-\frac{T-t}{T S_{t}^{2}} \frac{\partial U}{\partial \xi_{t}}, \quad \frac{\partial V}{\partial J_{t}}=\frac{t}{T J_{t}} \frac{\partial U}{\partial \xi_{t}} .
\end{aligned}
$$

Hence model (15) can be transformed into

$$
\begin{gathered}
\frac{\partial U}{\partial t}+\left(r_{t}-\frac{\tilde{\sigma}^{2}}{2}\right) \frac{T-t}{T} \frac{\partial U}{\partial \xi_{t}}+\frac{\widetilde{\sigma}^{2}}{2}\left(\frac{T-t}{T}\right)^{2} \frac{\partial^{2} U}{\partial \xi_{t}^{2}}-r_{t} U=0, \\
U\left(\xi_{T}, T\right)=\left(e^{\xi_{T}}-K\right)^{+} .
\end{gathered}
$$

Let

$$
\begin{gathered}
\tau=\gamma(t), \quad \eta_{\tau}=\xi_{t}+\alpha(t), \\
W\left(\tau, \eta_{\tau}\right)=U\left(t, \xi_{t}\right) e^{\beta(t)},
\end{gathered}
$$

which satisfies the conditions $\alpha(T)=\beta(T)=\gamma(T)=0$; then we have

$$
\begin{aligned}
& \frac{\partial U}{\partial t}=e^{-\beta(t)}\left(\frac{\partial W}{\partial \tau} \gamma^{\prime}(t)-\beta^{\prime}(t) W+\frac{\partial W}{\partial \eta_{\tau}} \alpha^{\prime}(t)\right), \\
& \frac{\partial U}{\partial \xi_{t}}=e^{-\beta(t)} \frac{\partial W}{\partial \eta_{\tau}}, \quad \frac{\partial^{2} U}{\partial \xi_{t}^{2}}=e^{-\beta(t)} \frac{\partial^{2} W}{\partial \eta_{\tau}^{2}} .
\end{aligned}
$$

Substituting (23) into (21), we have

$$
\begin{aligned}
\gamma^{\prime}(t) & \frac{\partial W}{\partial \tau}+\frac{\widetilde{\sigma}^{2}}{2}\left(\frac{T-t}{T}\right)^{2} \frac{\partial^{2} W}{\partial \eta_{\tau}^{2}}+\left[\left(r_{t}-\frac{\widetilde{\sigma}^{2}}{2}\right) \frac{T-t}{T}+\alpha^{\prime}(t)\right] \\
\times & \frac{\partial W}{\partial \eta_{\tau}}-\left(r_{t}+\beta^{\prime}(t)\right) W=0 .
\end{aligned}
$$


Let

$$
\begin{gathered}
\left(r_{t}-\frac{\tilde{\sigma}^{2}}{2}\right) \frac{T-t}{T}+\alpha^{\prime}(t)=0, \quad r_{t}+\beta^{\prime}(t)=0 \\
\gamma^{\prime}(t)=-\frac{\tilde{\sigma}^{2}}{2}\left(\frac{T-t}{T}\right)^{2}
\end{gathered}
$$

and combined with terminal conditions $\alpha(T)=\beta(T)=$ $\gamma(T)=0$, we can get

$$
\begin{aligned}
\alpha(t)= & \int_{t}^{T} r_{\theta}\left(\frac{T-\theta}{T}\right) d \theta-\int_{t}^{T} \frac{1}{2} \widetilde{\sigma}^{2}\left(\frac{T-\theta}{T}\right) d \theta \\
= & \int_{t}^{T} r_{\theta}\left(\frac{T-\theta}{T}\right) d \theta+\frac{H \sigma^{2}\left(T^{2 H+1}-t^{2 H+1}\right)}{T(2 H+1)} \\
& -\frac{\sigma^{2}\left(T^{2 H}-t^{2 H}\right)}{2} \\
& +\frac{1}{2} \operatorname{Le}(H) \sigma^{2} \frac{(T-t)^{2}}{T}-\frac{1}{2} \sigma^{2} b S_{t}(\delta t)^{2 H-1} \frac{(T-t)^{2}}{T} \\
\gamma(t)= & \int_{t}^{T} \frac{1}{2} \widetilde{\sigma}^{2}\left(\frac{T-\theta}{T}\right)^{2} d \theta \\
= & \frac{H}{T^{2}} \sigma^{2}\left(\frac{T^{2 H}-t^{2 H}}{2 H} T^{2}-2 T \frac{T^{2 H+1}-t^{2 H+1}}{2 H+1}\right. \\
& -\operatorname{Le}(H) \sigma^{2} \frac{(T-t)^{3}}{3 T^{2}}+b S_{t} \sigma^{2}(\delta t)^{2 H-1} \frac{(T-t)^{3}}{3 T^{2}} \\
\left.+\frac{T^{2 H+2}-t^{2 H+2}}{2 H+2}\right) & r_{\theta} d \theta .
\end{aligned}
$$

Thus (21) becomes

$$
\begin{gathered}
\frac{\partial W}{\partial \tau}=\frac{\partial^{2} W}{\partial \eta_{\tau}^{2}}, \\
W\left(\eta_{0}, 0\right)=\left(e^{\eta_{0}}-K\right)^{+} .
\end{gathered}
$$

According to the theory of classical heat conduction equation solution, we have

$$
\begin{aligned}
W\left(\eta_{\tau}, \tau\right) & =\frac{1}{2 \sqrt{\pi \tau}} \int_{\ln K}^{+\infty}\left(e^{y}-K\right) e^{-\left(\left(y-\eta_{\tau}\right)^{2} / 4 \tau\right)} d y \\
& =e^{\eta_{\tau}+\tau} N\left(\frac{2 \tau+\eta_{\tau}-\ln K}{\sqrt{2 \tau}}\right)-K N\left(\frac{\eta_{\tau}-\ln K}{\sqrt{2 \tau}}\right) .
\end{aligned}
$$

Let

$$
\begin{aligned}
r^{*}= & \frac{\int_{t}^{T} r_{\theta}((T-\theta) / T) d \theta}{T-t}-\frac{\sigma^{2}\left(T^{2 H}-t^{2 H}\right)}{2(T-t)} \\
& +\frac{H \sigma^{2}\left(T^{2 H+1}-t^{2 H+1}\right)}{T(2 H+1)(T-t)} \\
& +\frac{1}{2} \operatorname{Le}(H) \sigma^{2} \frac{T-t}{T}-\frac{1}{2} \sigma^{2} b S_{t}(\delta t)^{2 H-1} \frac{T-t}{T}, \\
\sigma^{*}= & \left(1-\frac{4 H\left(T^{2 H+1}-t^{2 H+1}\right)}{T(2 H+1)\left(T^{2 H}-t^{2 H}\right)}\right. \\
& +\frac{H\left(T^{2 H+2}-t^{2 H+2}\right)}{T^{2}(H+1)\left(T^{2 H}-t^{2 H}\right)} \\
& -2 \mathrm{Le}(H) \frac{(T-t)^{3}}{3 T^{2}\left(T^{2 H}-t^{2 H}\right)} \\
& +\frac{2 b S_{t}(\delta t)^{2 H-1}(T-t)^{3}}{3 T^{2}\left(T^{2 H}-t^{2 H}\right)} ;
\end{aligned}
$$

then

$$
\begin{aligned}
& \frac{2 \tau+\eta_{\tau}-\ln K}{\sqrt{2 \tau}} \\
& =\frac{\ln \left[\left(J_{t}^{t} S_{t}^{T-t}\right)^{1 / T} / K\right]+r^{*}(T-t)+\sigma^{* 2}\left(T^{2 H}-t^{2 H}\right)}{\sigma^{*} \sqrt{T^{2 H}-t^{2 H}}} \\
& \triangleq d_{1} \text {, } \\
& \frac{\eta_{\tau}-\ln K}{\sqrt{2 \tau}} \\
& =\frac{\ln \left[\left(J_{t}^{t} S_{t}^{T-t}\right)^{1 / T} / K\right]+r^{*}(T-t)}{\sigma^{*} \sqrt{T^{2 H}-t^{2 H}}} \\
& =d_{1}-\sigma^{*} \sqrt{T^{2 H}-t^{2 H}} \triangleq d_{2} \text {. }
\end{aligned}
$$

By variable restored, we obtain that

$$
\begin{aligned}
W & \left(\eta_{\tau}, \tau\right) \\
& =\left(J_{t}^{t} S_{t}^{T-t}\right)^{1 / T} e^{r^{*}(T-t)+\left(\sigma^{* 2} / 2\right)\left(T^{2 H}-t^{2 H}\right)} N\left(d_{1}\right)-K N\left(d_{2}\right) .
\end{aligned}
$$

So the value of geometric-average Asian call option on the time $t$ is

$$
\begin{aligned}
V\left(t, J_{t}, S_{t}\right)= & U\left(\xi_{t}, t\right)=W e^{-\beta(t)} \\
= & \left(J_{t}^{t} S_{t}^{T-t}\right)^{1 / T} e^{r^{*}(T-t)-\int_{t}^{T} r_{\theta} d \theta+\left(\sigma^{* 2} / 2\right)\left(T^{2 H}-t^{2 H}\right)} N\left(d_{1}\right) \\
& -K e^{-\int_{t}^{T} r_{\theta} d \theta} N\left(d_{2}\right) .
\end{aligned}
$$




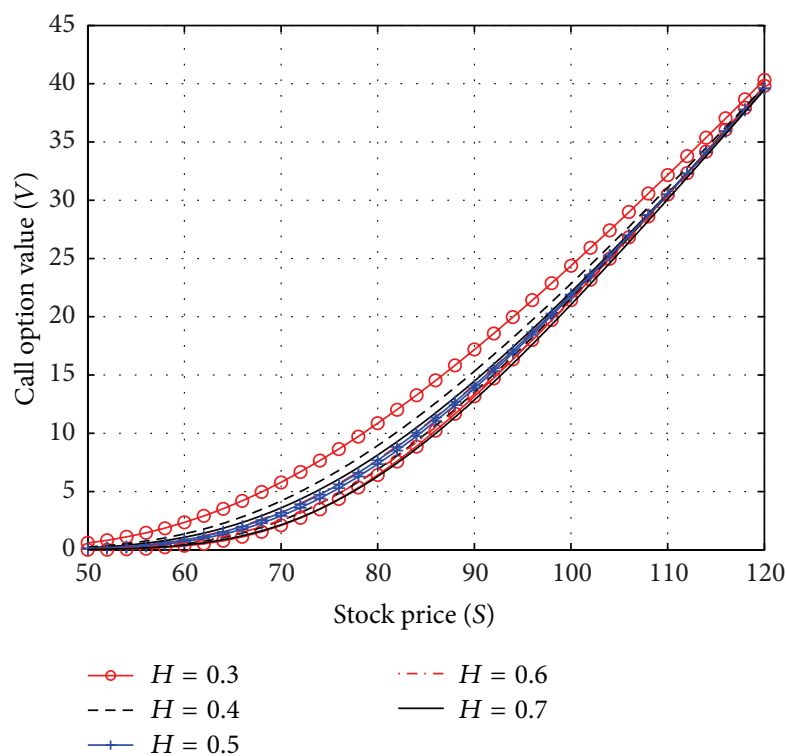

FIGURE 1: Asian call option pricing corresponding to the different $H$.

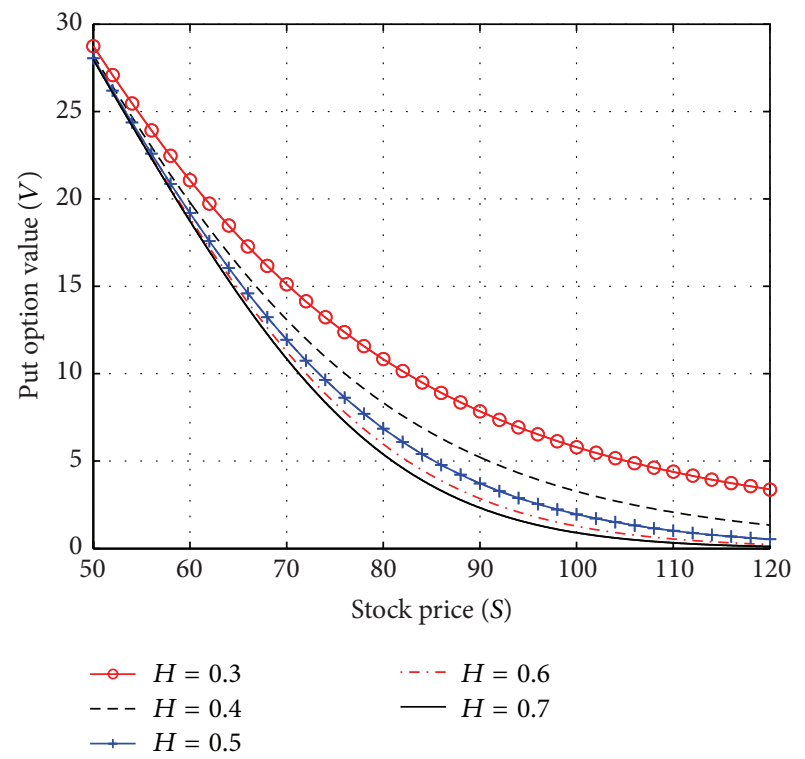

Figure 2: Asian put option pricing corresponding to the different $H$.

Corollary 4. If the riskless interest $r$ and the volatility $\sigma$ are constant, then the values $V_{C}$ and $V_{p}$ of Geometric-average Asian call option and put option respectively with monotonous transaction cost at time $t$ with expiration date $T$ and exercise price $K$ are

$$
\begin{aligned}
V_{C}\left(t, J_{t}, S_{t}\right)= & \left(J_{t}^{t} S_{t}^{T-t}\right)^{1 / T} e^{\left(r^{*}-r\right)(T-t)+\left(\sigma^{* 2} / 2\right)\left(T^{2 H}-t^{2 H}\right)} N\left(d_{1}\right) \\
& -K e^{-r(T-t)} N\left(d_{2}\right), \\
V_{P}\left(t, J_{t}, S_{t}\right)=- & \left(J_{t}^{t} S_{t}^{T-t}\right)^{1 / T} e^{\left(r^{*}-r\right)(T-t)+\left(\sigma^{* 2} / 2\right)\left(T^{2 H}-t^{2 H}\right)} N\left(-d_{1}\right) \\
+ & K e^{-r(T-t)} N\left(-d_{2}\right),
\end{aligned}
$$

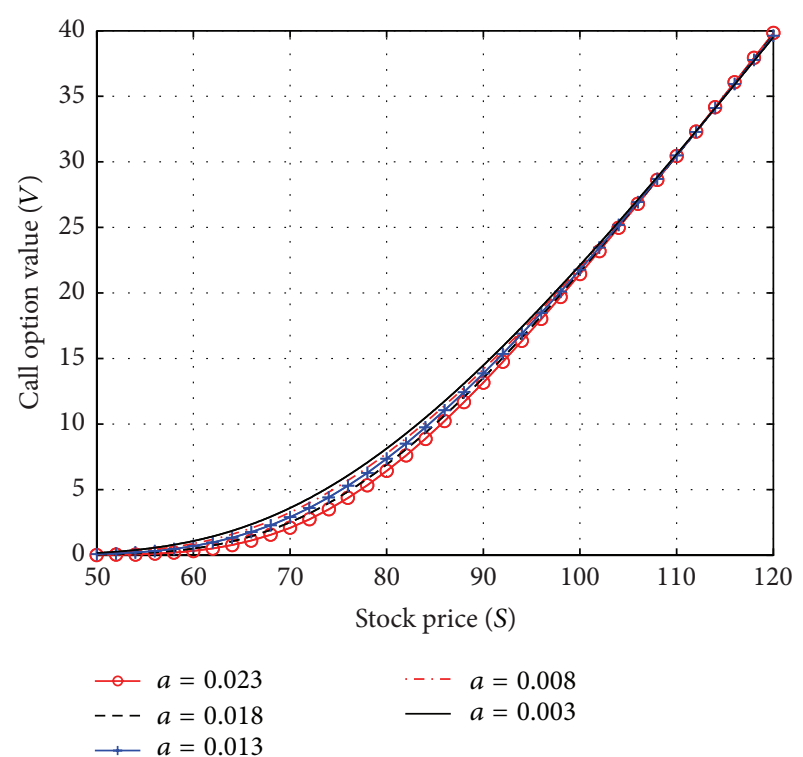

FIGURE 3: Asian put option pricing corresponding to the different $a$.

where

$$
\begin{aligned}
r^{*}= & \frac{r}{2 T}(T-t)-\frac{\sigma^{2}\left(T^{2 H}-t^{2 H}\right)}{2(T-t)}+\frac{H \sigma^{2}\left(T^{2 H+1}-t^{2 H+1}\right)}{T(2 H+1)(T-t)} \\
& +\frac{1}{2} L e(H) \sigma^{2} \frac{T-t}{T}-\frac{1}{2} \sigma^{2} b S_{t}(\delta t)^{2 H-1} \frac{T-t}{T}
\end{aligned}
$$

and the remaining symbols accord with Theorem 3.

\section{Numerical Experiments}

In this section, the influence of monotone transaction rate parameters and the Hurst exponent on Asian option value will be discussed through applying MATLAB software. The values of the parameters of geometric-average Asian options are assumed as follows:

$$
\begin{array}{ccc}
S_{t}=80, \quad t=0, & T=1, \quad r=0.05, \\
\sigma=0.4, \quad K=80, & H=0.5, \quad a=0.009, \\
b=0.002, \quad \delta t=0.02 . &
\end{array}
$$

With the option pricing formulas (33) presented, the value of the option can be calculated. Figures 1 and 2 give the relationships between the price of the underlying assets and the value of Asian call option and put option with different Hurst exponent. From the figures, Hurst exponent is inversely proportional to the value of Asian option. Figures 3 and 4 demonstrate the changes of Asian call option with the stock price under the different parameter $a$ and parameter $b$. We can draw such a conclusion: the option value increases with the parameter $b$ increasing and decreases with the parameter $a$ increasing. This is mainly because transaction cost rate is a decreasing function of $b$ and an increasing function of $a$. 


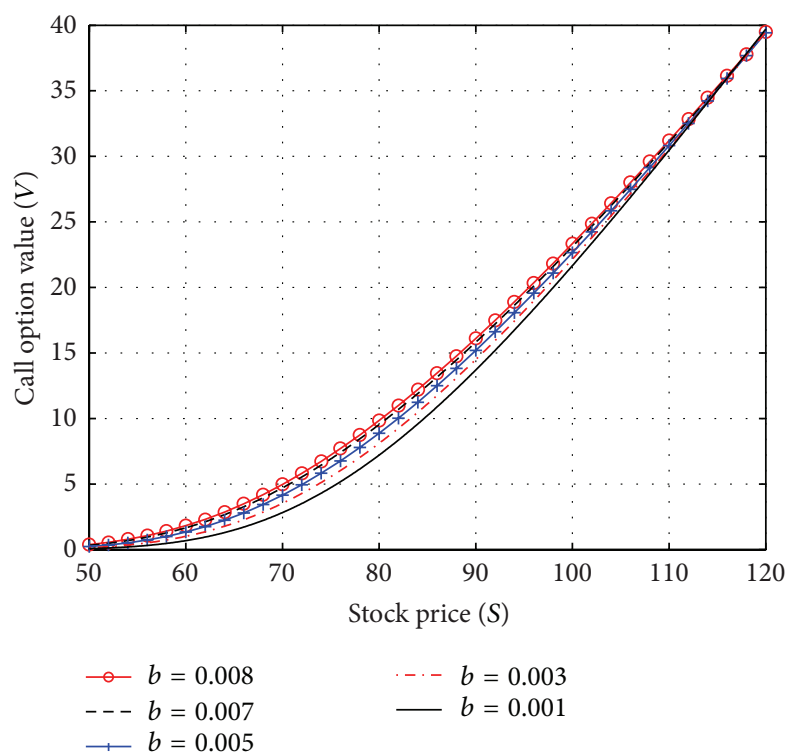

FIGURE 4: Asian put option pricing corresponding to the different $b$.

\section{Discussions and Conclusions}

In this paper, the problem of Asian option pricing with monotonous transaction cost rate under fractional Brownian motion was studied by using the portfolio technology and no arbitrage principle, and the pricing model was established. This model was solved by the method of partial differential equations, and the analytical expressions of the Asian option value were obtained. The numerical experiments showed that Hurst exponent of the fractional Brownian motion and transaction cost rates have a significant impact on the option value.

\section{Conflict of Interests}

The authors declare that they have no conflict of interests.

\section{Acknowledgment}

This work is supported by the Fundamental Research Funds for the Central Universities (2013XK03).

\section{References}

[1] E. E. Peters, "Fractal structure in the capital markets," Financial Analyst Journal, vol. 45, no. 4, pp. 32-37, 1989.

[2] T. E. Duncan, Y. Hu, and B. Pasik-Duncan, "Stochastic calculus for fractional Brownian motion. I. Theory," SIAM Journal on Control and Optimization, vol. 38, no. 2, pp. 582-612, 2000.

[3] F. Biagini, Y. Hu, B. Øksendal, and T. Zhang, Stochastic Calculus for Fractional Brownian Motion and Applications, 2008.

[4] E. Alos, O. Mazet, and D. Nualart, "Stochastic calculus with respect to the fractional Brownian motion," Stochastic Processes and Their Applications, vol. 86, pp. 121-139, 2000.

[5] C. Bender, "An Itô formula for generalized functionals of a fractional Brownian motion with arbitrary Hurst parameter,"
Stochastic Processes and their Applications, vol. 104, no. 1, pp. 81106, 2003.

[6] C. Necula, "Option pricing in a fractional Brownian motion environment," vol. 27, pp. 8079-8089, Academy of Economic Studies Bucharest, Romania, Preprint, Academy of Economic Studies, Bucharest, Romania, 2002.

[7] H. E. Leland, "Option pricing and replication with transactions costs," The Journal of Finance, vol. 40, no. 5, pp. 1283-1301, 1985.

[8] G. Barles and H. M. Soner, "Option pricing with transaction costs and a nonlinear Black-Scholes equation," Finance and Stochastics, vol. 2, no. 4, pp. 369-397, 1998.

[9] P. Amster, C. G. Averbuj, M. C. Mariani, and D. Rial, "A BlackScholes option pricing model with transaction costs," Journal of Mathematical Analysis and Applications, vol. 303, no. 2, pp. 688-695, 2005.

[10] H.-K. Liu and J.-J. Chang, "A closed-form approximation for the fractional Black-Scholes model with transaction costs," Computers \& Mathematics with Applications, vol. 65, no. 11, pp. 1719-1726, 2013.

[11] J. Wang, J.-R. Liang, L.-J. Lv, W.-Y. Qiu, and F.-Y. Ren, “Continuous time Black-Scholes equation with transaction costs in subdiffusive fractional Brownian motion regime," Physica A, vol. 391, no. 3, pp. 750-759, 2012.

[12] B. B. Mandelbrot, Fractals and Scaling in Finance: Discontinuity and Concentration, Springer, New York, NY, USA, 1997. 


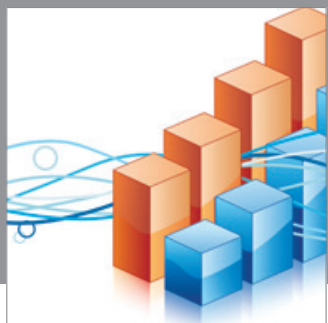

Advances in

Operations Research

mansans

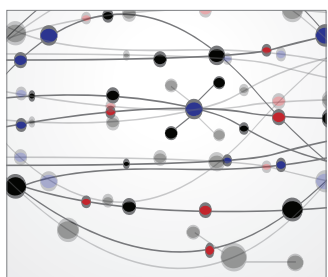

The Scientific World Journal
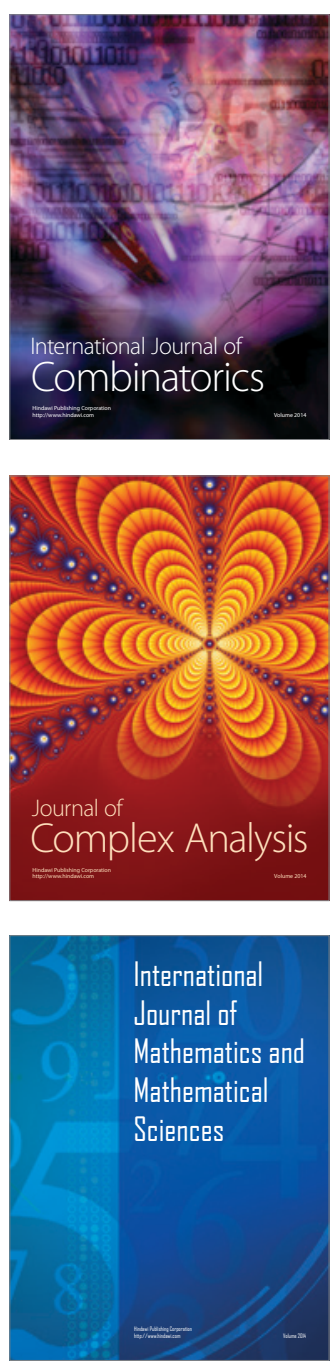
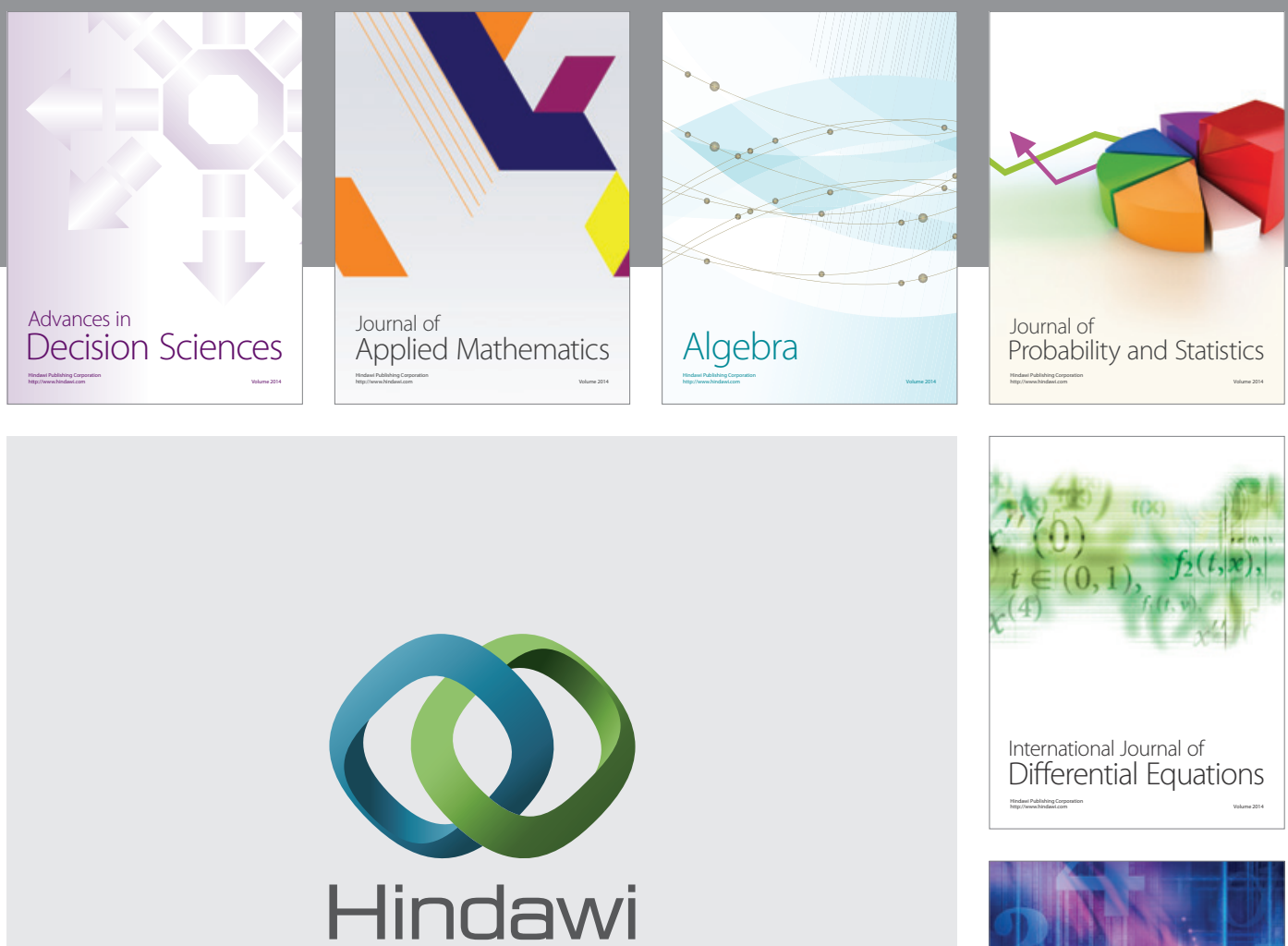

Submit your manuscripts at http://www.hindawi.com
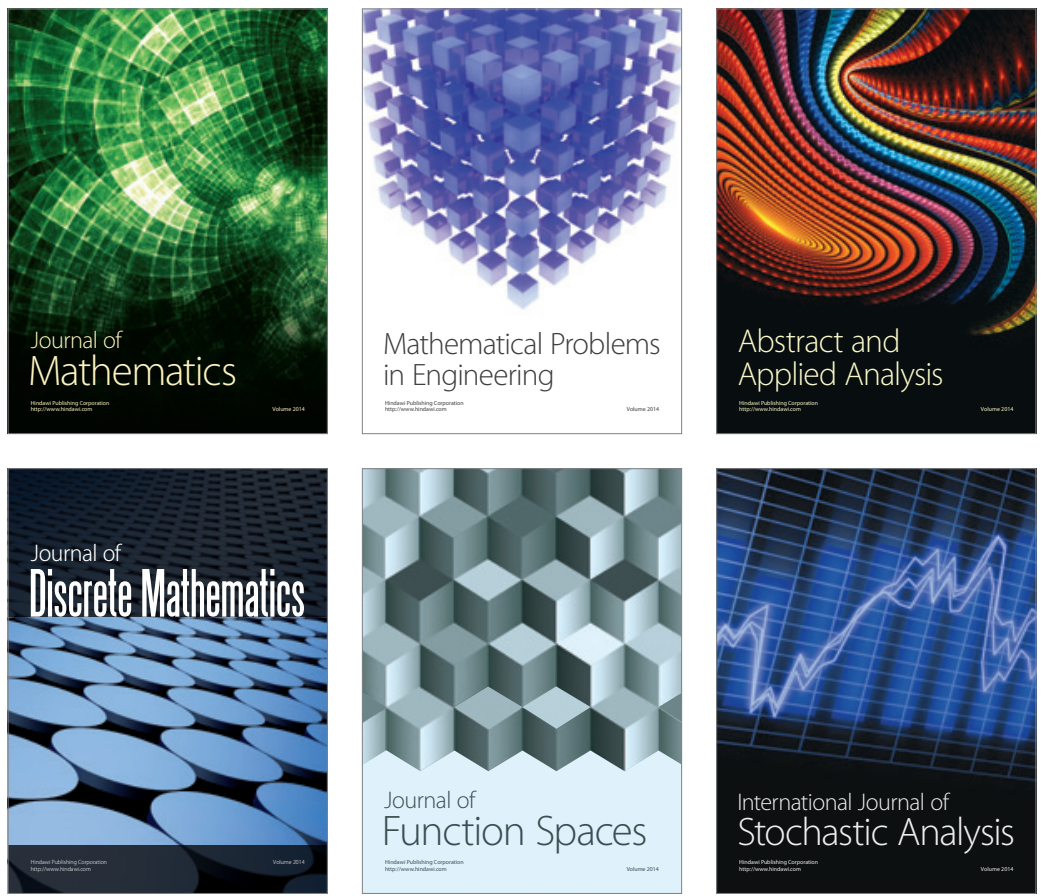

Journal of

Function Spaces

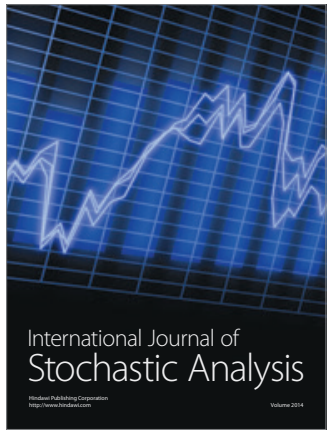

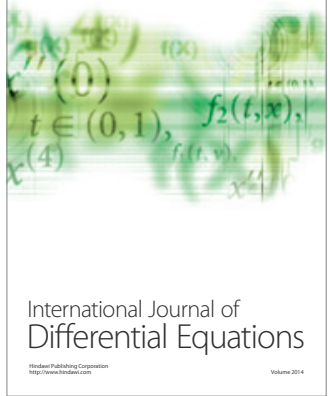
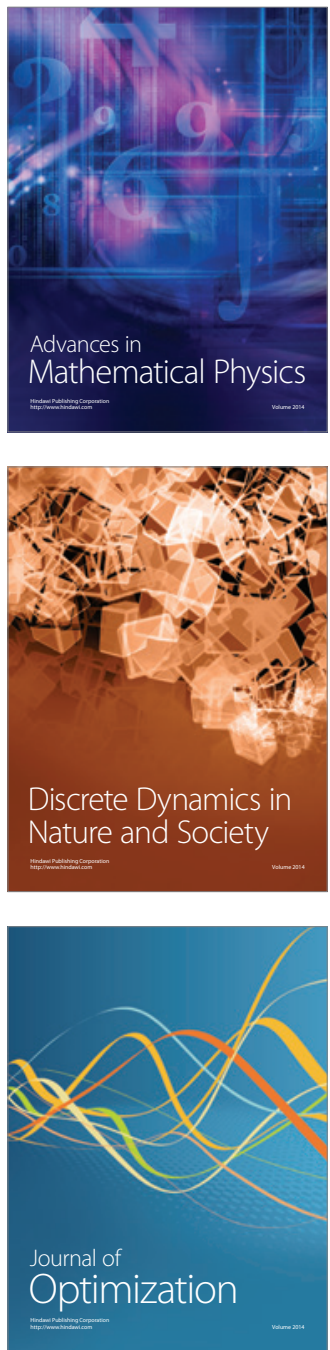